\title{
Pulmonary fibrosis linked to variants in the $A C D$ gene, encoding the telomere protein TPP1
}

\author{
To the Editor:
}

Telomeres are repetitive DNA sequences at the ends of chromosomes. Telomeres shorten with every cell division, and thus become increasingly short with age. Stable telomeres are necessary for cellular survival, and critically short or dysfunctional telomeres lead to cellular senescence or apoptosis. Mutations in genes encoding telomere-associated proteins can lead to increased telomere shortening or telomere dysfunction [1]. These mutations can cause various disease manifestations, which are termed telomere syndromes [1].

Disease-causing mutations in genes encoding telomere-associated proteins, most commonly TERT, TERC, RTEL1 and PARN, have been identified in up to $11 \%$ of patients with sporadic idiopathic pulmonary fibrosis (IPF), and in up to $25 \%$ of patients with familial pulmonary fibrosis (two or more first-degree relatives with pulmonary fibrosis) [2-4]. Here we studied the $A C D$ gene, which encodes the telomere protein TPP1, in patients with pulmonary fibrosis.

Between October 2017 and December 2018, 60 unrelated patients with pulmonary fibrosis from St Antonius Hospital (Nieuwegein, the Netherlands), a referral centre for patients with interstitial lung diseases, underwent genetic screening. Screening was offered in case of a family history of pulmonary fibrosis, clinical suspicion of a telomere syndrome or relatively young age at presentation $(\mathrm{n}=44, \mathrm{n}=9$ and $\mathrm{n}=7$, respectively). Clinical suspicion of a telomere syndrome was based on haematological abnormalities such as bone marrow failure or anaemia in six patients and liver cirrhosis in three patients. The cohort included 18 females, and the median age at the time of genetic screening was 69 years (range 33-84 years).

DNA was isolated from blood samples. Exome sequencing and processing of the data were performed at the University Medical Center Utrecht. After enrichment of the exome with the Agilent SureSelect CREV2 kit (Agilent Technologies, Santa Clara, CA, USA), whole-exome sequencing was performed on a Novaseq 6000 sequencer (Illumina, San Diego, CA, USA). The Illumina sequencing data was processed with the in-house pipeline, IAP v2.6.1 [5], including GATK v3.4-46, according to best-practice guidelines [6]. Results were filtered for exonic variants with a population frequency $<0.5 \%$ in 36 genes related to telomere syndromes or pulmonary fibrosis (ABCA3, ACD, AP3B1, CSF2RA, CSF2RB, CTC1, DKC1, FAM111B, HPS1, HPS4, ITGA4, LIG4, MARS, NAF1, NKX2-1, NOP10, PARN, POT1, RNF168, RTEL1, SAMD9L, SFTPA1, SFTPA2, SFTPB, SFTPC, SFTPD, STN1, TEN1, TERC, TERF1, TERF2, TERT, TINF2, TMEM173, USB1 and WRAP53). Leukocyte telomere length was measured using previously described methods [7]. Briefly, telomere length was estimated for each sample from the ratio of telomere repeat copy number to a single gene (human $\beta$-globin gene) copy number (T/S ratio). Measurements were performed on the Bio-Rad CFX96 ${ }^{\text {TM }}$ Real-Time PCR Detection System (Bio-Rad, Hercules, CA, USA). Reference values were derived from a cohort of 164 healthy controls. Pulmonary fibrosis diagnoses were made after multidisciplinary discussion, in accordance with the 2018 American Thoracic Society/European Respiratory Society/Japanese Respiratory Society/Latin American Thoracic Society guideline [8]. All patients gave written informed consent in research project R05-08A.

In 19 patients, potentially disease-causing variants in one or more telomere genes were found, including four patients with pathogenic mutations in TERT and four patients with pathogenic mutations in RTEL1. Potentially disease-causing variants in $A C D$ were identified in three patients (figure 1). The first patient

$@$ ERSpublications

Pulmonary fibrosis is associated with mutations in the $A C D$ gene, encoding the telomere protein TPP1. Potentially disease-causing variants were found in $5 \%$ of a cohort of 60 unrelated patients with pulmonary fibrosis who were referred for genetic screening. http://bit.ly/2Mw9 $\mathrm{Cn} 0$

Cite this article as: Hoffman TW, van der Vis JJ, van der Smagt JJ, et al. Pulmonary fibrosis linked to variants in the ACD gene, encoding the telomere protein TPP1. Eur Respir J 2019; 54: 1900809 [https:// doi.org/10.1183/13993003.00809-2019]. 
a)

) Patient

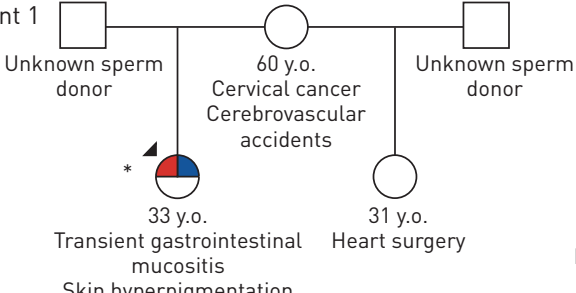

Skin hyperpigmentation
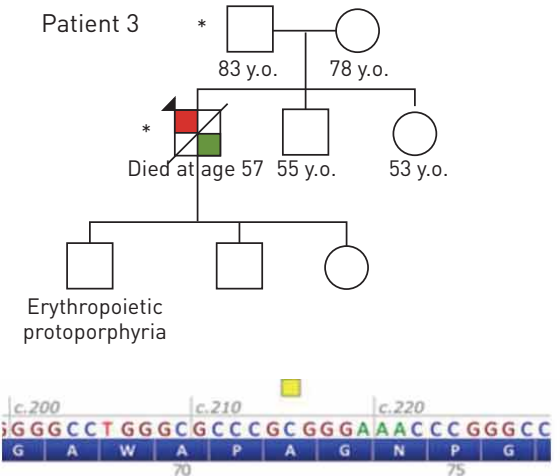

:CCCGGACCCGCGGGCGCCCT TTGGGCCCGG
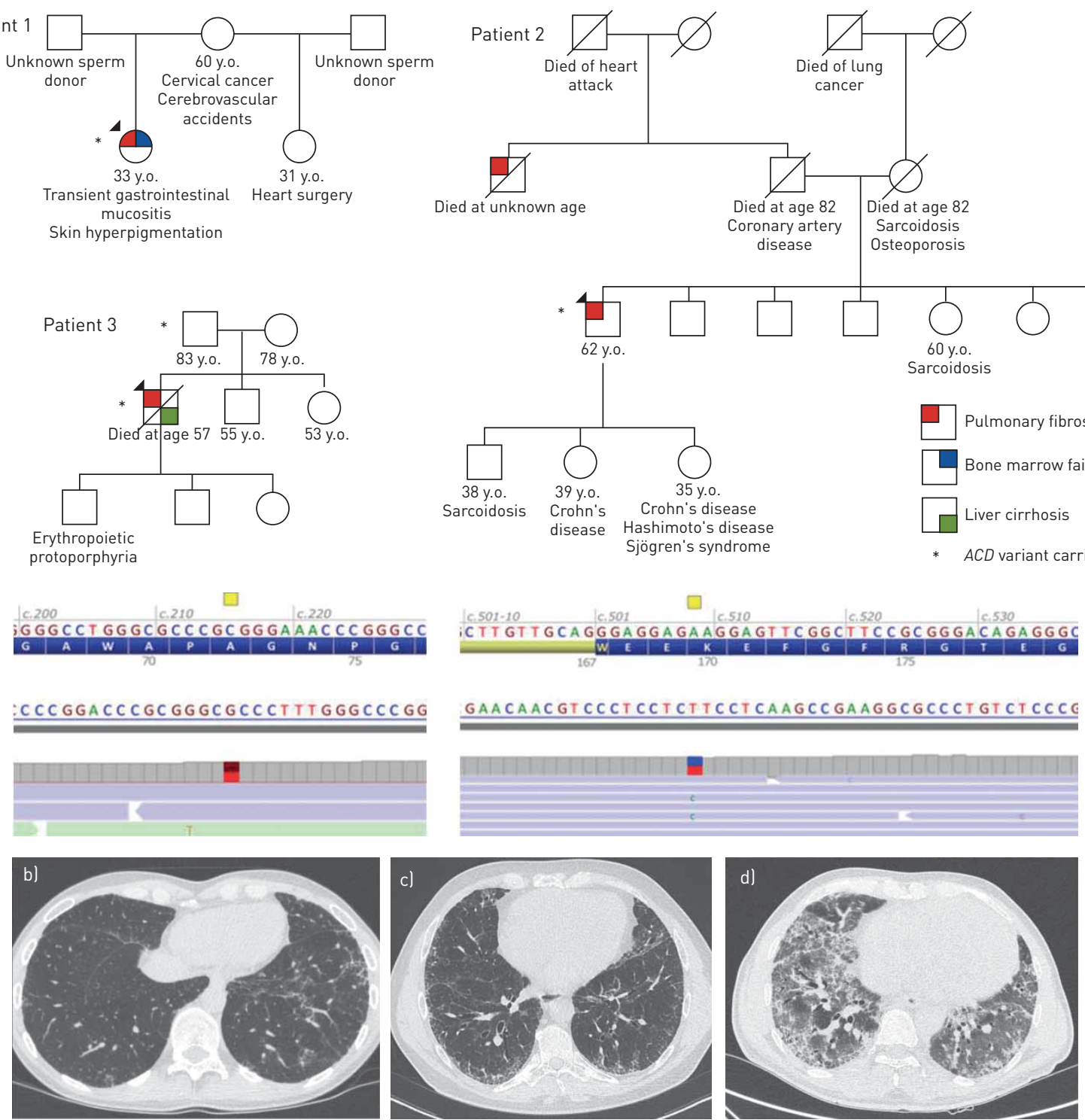

\section{GAACAACGTCCCTCCTCTTCCTCAAGCCGAAGGCGCCCTGTCTCCCG}
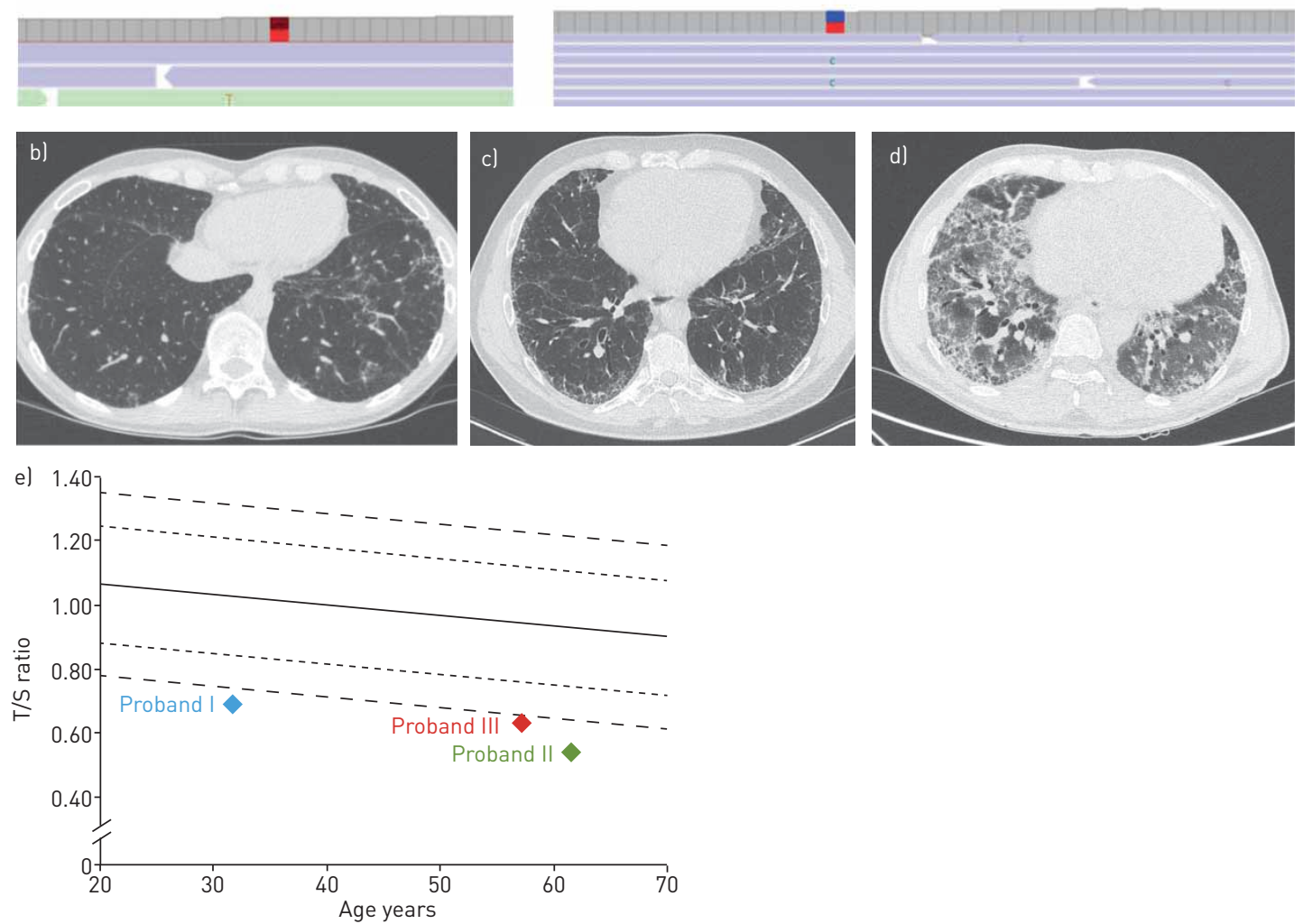

FIGURE 1 a) Pedigrees for patients 1-3. Sequencing data are shown below the pedigree for patients 2 and 3 . b-d) High-resolution computed tomography (HRCT) scans of the chest were graded based on the 2018 Joint IPF Diagnostic Guideline Statement [8]. HRCTs for patients 1-3 show pulmonary fibrosis in the lower lobes. b) In patient 1, there was bilateral subpleural reticulation and honeycombing, as well as minor traction bronchiectasis in the left lung. The pattern was inconsistent with a usual interstitial pneumonia (UIP) pattern because of central extension of the fibrosis as well as airtrapping (not shown). c) In patient 2, the HRCT showed subpleural reticulation, predominantly in the lung bases, but without honeycombing, indicating a possible UIP pattern. d) In patient 3, there was subpleural reticulation with traction bronchiectasis predominantly in the lower lobes, as well as honeycombing, consistent with a UIP pattern. Ground-glass opacities were noted, but this was not the dominant pattern. e) Leukocyte telomere length in patients 1-3 compared to a cohort of 164 healthy controls. The uninterrupted line represents the 50th percentile for age. The dashed lines represent the 99th, 90th, 10th and 1st percentiles for age. *: ACD variant carrier. 
was a 33-year-old female referred to our centre because of dyspnoea on exertion. She had been diagnosed with bone marrow failure at age 25 years and had experienced several transient episodes of gastrointestinal mucositis, which had not led to a definitive diagnosis, and had improved without specific treatment. She reported having grey hair since the age of 21 years. The family history was only available for her mother and sister, and was not suggestive of a telomere syndrome (figure 1a). She had a smoking history of 8 pack-years, but no other fibrogenic exposures. On physical examination, reticular hyperpigmentation of the skin on the neck was noted, but no oral leukoplakia or nail dystrophy. No hepatic abnormalities were seen on abdominal ultrasound. A high-resolution computed tomography (HRCT) scan of the thorax showed pulmonary fibrosis with honeycombing, but inconsistent with a usual interstitial pneumonia (UIP) pattern (figure 1b). The patient was given a working diagnosis of pulmonary fibrosis in the context of a telomere syndrome. Genetic analysis revealed a heterozygous deletion in ACD: c.508-510del; p.(Lys170del) (NM_001082486.1), and no abnormalities in the other tested genes.

The second patient was a 62-year-old male who presented with dyspnoea on exertion. His past medical history revealed no clues suggestive of telomere disease, and he reported no early greying of his hair. His family history was notable for pulmonary and autoimmune diseases in several family members, including pulmonary fibrosis in an uncle (figure 1a). The patient did not report any significant fibrogenic exposures and had a smoking history of 2 pack-years when he was in his twenties. No skin abnormalities were noted on physical examination, and laboratory investigations revealed normal platelet, erythrocyte and leukocyte counts and no macrocytosis. Pulmonary fibrosis with a probable UIP pattern (figure 1c) was seen on HRCT, and the patient was given a working diagnosis of IPF. Genetic screening revealed a heterozygous variant in $A C D$ : c.508A>G; p.(Lys170Glu), and no abnormalities in the other tested genes.

The third patient was a 57-year-old male who presented with dyspnoea on exertion and chronic cough. His past medical history was notable for cryptogenic liver cirrhosis since the age of 24 years, and he reported grey hair since age 30 years. His family history revealed no additional clues for a telomere syndrome (figure 1a). The patient had never smoked, but he worked as an electrician and reported exposure to rockwool and dust at work. On physical examination, vitiligo of his fingers, toes and right lower leg was seen, but no other skin abnormalities were noted. Laboratory investigations revealed mild thrombocytopenia, with normal erythrocyte and leukocyte counts and no macrocytosis. Pulmonary fibrosis with honeycombing, consistent with a UIP pattern (figure 1d), was seen on HRCT. The patient was given a diagnosis of IPF. Genetic screening revealed a heterozygous variant in ACD: c.215C>A; p.(Ala72Glu), and no abnormalities in the other tested genes.

Leukocyte telomere length was below the first percentile for age in all three patients (figure 1e). Blood samples were not available for the family members of patients 1 and 2 . However, genetic analysis could be performed in the parents of patient 3, and the patient's 82-year-old father was found to have the same $A C D$ variant. The father had no signs or symptoms suggestive of telomere disease, but did not undergo additional investigations such as lung function testing or computed tomography scanning.

This study shows that pulmonary fibrosis patients can have heterozygous mutations in $A C D$. In $5 \%$ of patients with pulmonary fibrosis referred for genetic screening, (potentially) disease-causing variants were present. $A C D$ encodes the shelterin component TPP1, which is responsible for telomerase recruitment to telomeres [9]. Disease-causing mutations in ACD have been identified previously in patients with aplastic anaemia and Hoyeraal-Hreidarsson syndrome, the most severe form of dyskeratosis congenita [10, 11].

The variants that were identified in patients 1 and 2 are located in the oligonucleotide/oligosaccharide-binding (OB)-fold domain of TPP1, which is the telomerase-interacting domain. The variant identified in patient 3 is located in the N-terminal domain, the function of which is currently not known. The variant in patient 1 is classified as a pathogenic mutation, as the same variant has previously been functionally characterised in patients with aplastic anaemia and Hoyeraal-Hreidarsson syndrome [10-12]. This showed that it disrupts TPP1 binding to telomerase, and the mutation itself is sufficient for causing telomere dysfunction in a human cell line [13]. The phenotype of patient 1 is of interest because it fits well within the concept of a telomere syndrome, but is not a classic case of dyskeratosis congenita despite the young age at presentation and involvement of multiple organs. Because of the extremely short telomeres in patients 2 and 3 , as well as liver cirrhosis and early greying consistent with a telomere syndrome phenotype in patient 3 , a telomere gene mutation would be a likely explanation for their disease. The $A C D$ variants from patient 2 and 3 were absent in the Exome Sequencing Project, 1000 Genomes Project and the Exome Aggregation Consortium. Furthermore, PolyPhen-2 analysis (at http://genetics.bwh.harvard.edu/pph2/index.shtml) predicted both variants to be probably damaging. Of note, it has been suggested that the quantitative PCR technique is less reliable for measuring telomere length than other techniques [14].

The variant in patient 2 is classified as probably pathogenic [12], because it concerns the same amino acid, Lys170, as the pathogenic mutation in patient 1 . The variant in patient 3 is classified as a variant of unknown significance [12]. The fact that the father of patient 3 was healthy, despite carrying the same 
$A C D$ variant, would suggest that the variant is not disease-causing. Alternatively, genetic anticipation, where symptoms of a telomere syndrome become more severe and have an earlier onset in each successive generation, occurs in families with telomere syndrome. This allows for incomplete penetrance of telomere syndrome features in the father [1]. Unfortunately, there was no blood sample available for telomere length measurement.

In conclusion, this study shows that pulmonary fibrosis is associated with mutations in the $A C D$ gene, encoding the telomere protein TPP1. Potentially disease-causing variants were found in $5 \%$ of the patients in this cohort, including two mutations classified as pathogenic (3\% of patients).

Thijs W. Hoffman ${ }^{1}$, Joanne J. van der Vis ${ }^{1,2}$, Jasper J. van der Smagt ${ }^{3}$, Maarten P.G. Massink ${ }^{3}$, Jan C. Grutters ${ }^{1,4}$ and Coline H.M. van Moorsel ${ }^{1,4}$

${ }^{1}$ Dept of Pulmonology, Interstitial Lung Diseases Center of Excellence, St Antonius Hospital, Nieuwegein, The Netherlands. ${ }^{2}$ Dept of Clinical Chemistry, St Antonius Hospital, Nieuwegein, The Netherlands. ${ }^{3}$ Dept of Genetics, University Medical Center Utrecht, Utrecht, The Netherlands. ${ }^{4}$ Division of Heart and Lungs, University Medical Center Utrecht, Utrecht, The Netherlands.

Correspondence: Coline H.M. van Moorsel, Dept of Pulmonology, St Antonius Hospital, Koekoekslaan 1, 3435 CM, Nieuwegein, The Netherlands. E-mail: c.van.moorsel@antoniusziekenhuis.nl

Received: 23 April 2019 | Accepted after revision: 17 Aug 2019

Acknowledgements: We thank K.M. Kazemier (Dept of Pulmonology, St Antonius Hospital, Nieuwegein, the Netherlands) for her expert assistance with performing telomere length measurement, H.W. van Es (Dept of Radiology, St Antonius Hospital) for his expert interpretation of the radiology images, and A.E. Schoemaker (Dept of Genetics, University Medical Center Utrecht, Utrecht, the Netherlands) for her assistance with collecting patient information.

Conflict of interest: T.W. Hoffman has nothing to disclose. J.J. van der Vis reports grants from ZonMW, during the conduct of the study. J.J. van der Smagt has nothing to disclose. M.P.G. Massink has nothing to disclose. J.C. Grutters reports grants from ZonMW, during the conduct of the study. C.H.M. van Moorsel reports grants from ZonMW, during the conduct of the study.

Support statement: This research was enabled by ZonMW TopZorg St Antonius Science Corner grant (grant number 842002003; www.zonmw.nl; J.J. van der Vis, J.C. Grutters and C.H.M. van Moorsel). Funding information for this article has been deposited with the Crossref Funder Registry.

\section{References}

Armanios M, Blackburn EH. The telomere syndromes. Nat Rev Genet 2012; 13: 693-704.

2 Petrovski S, Todd JL, Durheim MT, et al. An exome sequencing study to assess the role of rare genetic variation in pulmonary fibrosis. Am J Respir Crit Care Med 2017; 196: 82-93.

3 Borie R, Tabèze L, Thabut G, et al. Prevalence and characteristics of TERT and TERC mutations in suspected genetic pulmonary fibrosis. Eur Respir J 2016; 48: 1721-1731.

4 Kannengiesser C, Borie R, Ménard C, et al. Heterozygous RTEL1 mutations are associated with familial pulmonary fibrosis. Eur Respir J 2015; 46: 474-485.

5 Ernst RF, Van Roosmalen M, De Ligt J, et al. UMCUGenetics IAP: V2.6.1. 2017. http://doi.org/10.5281/zenodo. 1040130.

6 Van der Auwera GA, Carneiro MO, Hartl C, et al. From FastQ data to high confidence variant calls: the Genome Analysis Toolkit best practices pipeline. Curr Protoc Bioinformatics 2013; 43: 11.10.1-11.10.33.

7 Snetselaar R, van Moorsel CH, Kazemier KM, et al. Telomere length in interstitial lung diseases. Chest 2015; 148: 1011-1018.

8 Raghu G, Remy-Jardin M, Myers JL, et al. Diagnosis of idiopathic pulmonary fibrosis. an official ATS/ERS/JRS/ ALAT clinical practice guideline. Am J Respir Crit Care Med 2018; 198: e44-e68.

9 Rajavel M, Mullins MR, Taylor DJ. Multiple facets of TPP1 in telomere maintenance. Biochim Biophys Acta 2014; 1844: 1550-1559.

10 Guo Y, Kartawinata M, Li J, et al. Inherited bone marrow failure associated with germline mutation of ACD, the gene encoding telomere protein TPP1. Blood 2014; 124: 2767-2774.

11 Kocak H, Ballew BJ, Bisht $\mathrm{K}$, et al. Hoyeraal-Hreidarsson syndrome caused by a germline mutation in the TEL patch of the telomere protein TPP1. Genes Dev 2014; 28: 2090-2102.

12 Richards S, Aziz N, Bale S, et al. Standards and guidelines for the interpretation of sequence variants: a joint consensus recommendation of the American College of Medical Genetics and Genomics and the Association for Molecular Pathology. Genet Med 2015; 17: 405-424.

13 Bisht K, Smith EM, Tesmer VM, et al. Structural and functional consequences of a disease mutation in the telomere protein TPP1. Proc Natl Acad Sci USA 2016; 113: 13021-13026.

14 Khincha PP, Dagnall CL, Hicks B, et al. Correlation of leukocyte telomere length measurement methods in patients with dyskeratosis congenita and in their unaffected relatives. Int J Mol Sci 2017; 18: E1765. 